\title{
Methods to Improve Energy Conversion Efficiency of Dielectric Elastomer Generators
}

\author{
Hengtong Cheng ${ }^{1,2, a}$, Zhenqiang Song ${ }^{1, b}$, Shijie Zhu $^{1, \mathrm{c}}$ and Kazuhiro Ohyama ${ }^{1}$ \\ ${ }^{1}$ Department of Intelligent Mechanical Engineering, Graduate School of Fukuoka Institute of \\ Technology, Japan \\ ${ }^{2}$ Department of Aerospace Engineering, Graduate School of Nanjing University of Science and \\ Technology, China \\ amcm18104@bene.fit.ac.jp, ’ zqsong33@126.com, 'chu@fit.ac.jp.
}

\begin{abstract}
Keywords: Dielectric elastomer generators, Energy conversion efficiency, Energy density,
\end{abstract} Pre-stretch ratio

\begin{abstract}
Dielectric elastomer generators (DEGs) are based on the electromechanical response of the dielectric elastomer film sandwiched between the compliant electrodes on each side, which are capable of converting mechanical energy from diverse sources (e.g, ocean wave) into electrical energy. In essence, DEG is a voltage up-converter using mechanical energy to increase the electrical energy of the charge on a soft capacitor. We evaluated the effect of input voltage and pre-stretch ratio on the energy conversion efficiency of DEG. With a power supply of $2.2 \mathrm{kV}$ and a pre-stretch ratio of 2, the maximum net electrical energy density and energy conversion efficiency in a single harvesting cycle were measured to be $413 \mathrm{~J} / \mathrm{kg}$ and $15.8 \%$, respectively. The experimental results show that, with the higher input voltage and the larger stretch ratio range, higher the energy conversion efficiency of DEG can be achieved.
\end{abstract}

\section{Introduction}

With the development of science and technology, the demand for electricity has increased dramatically [1]. We can already obtain electrical energy from hydropower, wind, solar photovoltaic and tide. However, other sources of mechanical energy, such as human movement, flowing water, mechanical vibration, and ocean waves, have remained untapped [2,3]. Dielectric elastomer (DE) is a kind of electroactive polymers with the extraordinary deforming ability [1-9]. Dielectric elastomer generators (DEGs) have emerged as a viable and promising candidate a few years ago. Dielectric elastomer has obtained much attention due to their flexibility, large deformation, high energy density and high energy conversion efficiency. In addition, it is made from low-cost materials that can be easily fabricated in large-area sheets. As a new energy transduction technology, DEGs are capable of converting mechanical energy from diverse sources into electrical energy. The DE film is stretched and charged firstly. Then, under the open-circuit condition, the tensile force of the film is removed, and the film thickness increases, while the area of the film decreases, which increase the voltage on the film. Experiments have shown that the energy density of DEGs can be up to $780 \mathrm{~J} / \mathrm{kg}$ [1], which is at least an order of magnitude higher than the specific energies of piezoelectric ceramics and electromagnetic generators.

The maximum amount of energy that can be harvested from a DEG is constrained by electrical breakdown (EB), electromechanical instability (EMI), loss of tension and rupture by excessive stretching $[1,3,8]$. The diagram was redrawn in Fig.1 for DE elastomers under equi-biaxial tension loading [1,6]. Under the same stretches, the net energy density of triangle cycles is higher than that of quadrangle cycles due to the larger enclosed area [6]. The triangle cycles were designed for maximizing the energy density and energy conversion efficiency. The proposed electromechanical 
harvesting scheme is shown by the triangle A-B-C-A on the voltage-charge work-conjugate plane. We can conclude that a desirable energy harvesting cycle of the DEG should cover this enclosed area as much as possible.

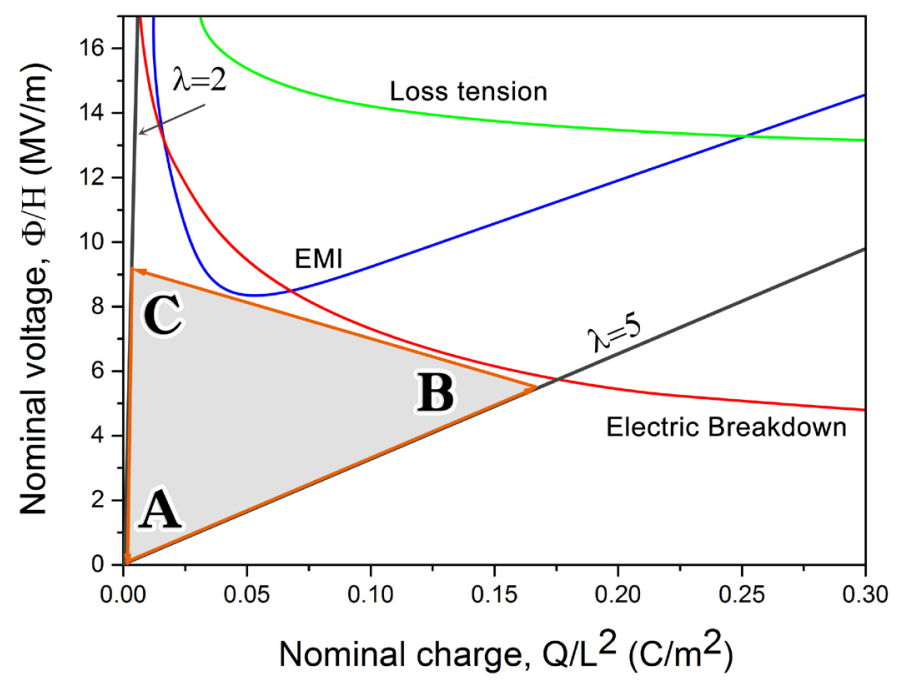

Fig. 1 Electromechanical energy conversion cycles defined in planes of work-conjugate coordinates, bounded by electrical breakdown, electromechanical instability and loss of tension.

In 2001, DEGs were first proposed for the electrical energy harvesting [1]. In recent ten years, although various experimental models using different materials and harvest circuits have been reported in the literature, compared with the theoretical calculation $1700 \mathrm{~J} / \mathrm{kg}[5,7]$, the energy density and energy conversion efficiency are still far less than the theoretical prediction. Moreover, the energy harvesting performance is strongly affected by the charge leakage and the viscosity of the acrylic elastomer [1-9]. The purpose of this paper is to find a method for increasing energy density and energy conversion efficiency by studying the effects of pre-stretch ratio and input voltage on energy density and energy conversion efficiency.

\section{Material and Experimental Procedures}

The VHB 4905 acrylic elastomer (3M Co., Ltd.) was used as experimental material. Carbon conductive grease (MG Chemicals CAT.NO.846-80G) was pasted on both sides to serve as compliant electrodes. The experimental film thickness, $H$, was $0.5 \mathrm{~mm}$. $L$ is the thickness when the experimental film is not deformed and the electrode diameter, $D$, was $40 \mathrm{~mm}$. The mass of the electrode-pasted part of the film, $M$, was $0.60 \mathrm{~g}$. The applied force, $F$, was provided by a linear motor actuator. The equi-biaxial tension loading was accomplished by applying radial forces to the circumference of the DE film through a combination of wires, clips, pulleys and wooden bracket. The movement of servomotor and data acquisition were performed with LabVIEW software.

In the electromechanical cycles, the energy harvesting tests were conducted with the pre-stretch ratios of 2, 2.5, 3 and 3.5, while the maximum stretch ratio was set to be 5 . The nominal input voltages were chosen to be $1.8 \mathrm{kV}, 2 \mathrm{kV}$ and $2.2 \mathrm{kV}$ with a constant charging current of $300 \mu \mathrm{A} . R$ is the radius of the film at the stretched state. $R_{0}$ is the initial state radium and $\lambda$ is defined as the ratio of the stretched state to the initial state in the direction of the stretching force. 

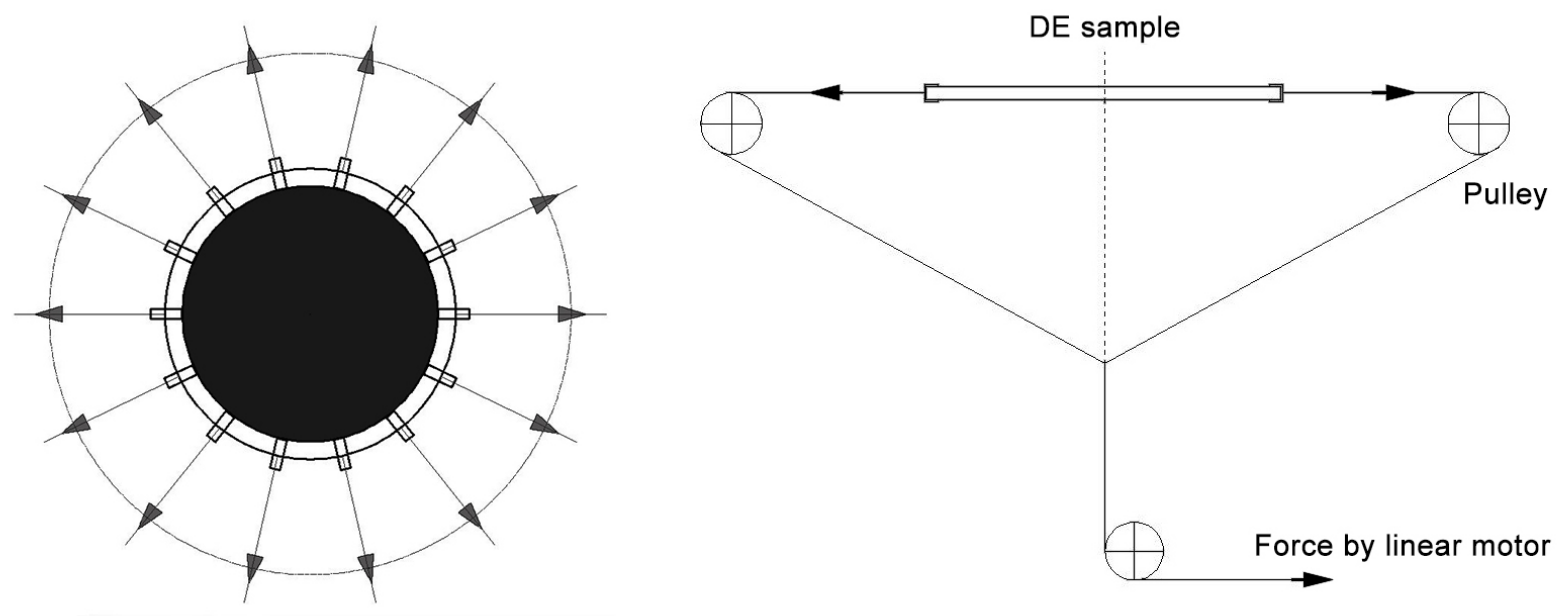

Fig. 2 Schematic of the equi-biaxial mechanical experimental device. The elastomer was coated with conductive carbon grease on both sides as compliant electrodes.

In essence, DEG is a mechano-capacitive device that boosts charge from low voltage to high voltage. The power generation cycle of DEG is divided into 4 phases. (1) The elastomer was stretched to a workable maximum stretch ratio $(\lambda=5)$ where lower than its rupture value. (2) An input voltage from the power supply was applied to charge the DE. (3) The mechanical loading was removed; due to the effects of Maxwell pressure, some residual strain may still be presented. (4) The charges were then harvested at high voltage.

\section{Results and Discussion}

In the $\mathrm{A}_{0}$-A phase, DE film begins to be stretched and deformed. Mechanical energy is converted into elastic potential energy stored in the DE elastomer. When state A is reached, the membrane got to its maximum workable stretch. In order to prevent the sample from being damaged, we choose the largest workable stretch ratio of $\lambda=5$. Throughout the stretching process, the DE membrane has no charge on its both sides and switch $\mathrm{S}_{1}$ and switch $\mathrm{S}_{2}$ are inactivated from state $\mathrm{A}_{0}$ to state $\mathrm{A}$.

In the A-B phase, this process charging the DE film with a constant current. Switch $\mathrm{S}_{1}$ is activated when the film is stretched to the maximum ratio. When the voltage of the DE membrane is equal to the supply power, the switch $\mathrm{S}_{1}$ automatically disconnects and stops charging.

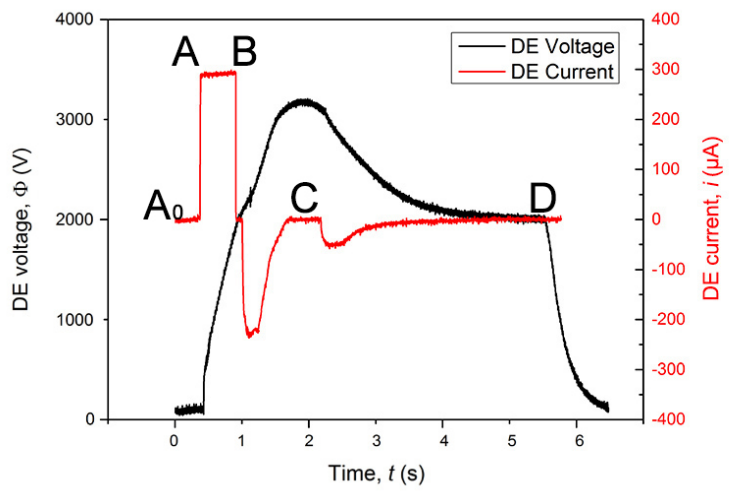

(a)

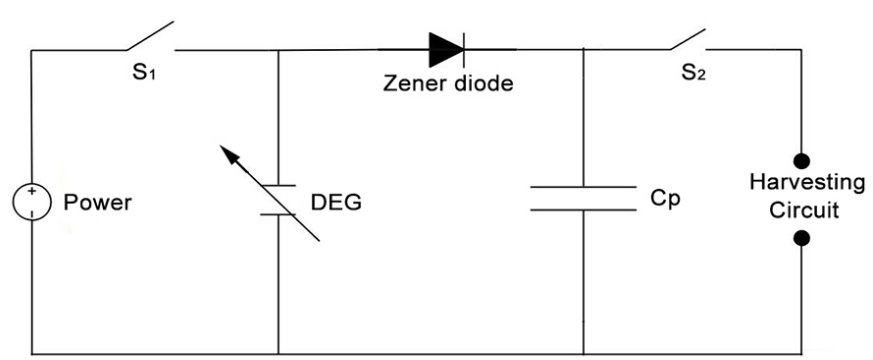

(b)

Fig. 3 (a) Voltage and current as a function of time during a single harvesting cycle,

(b) Electrical circuit showing energy harvesting principle. 
In the $\mathrm{B}-\mathrm{C}$ phase, switches $\mathrm{S}_{1}$ and $\mathrm{S}_{2}$ are inactivated during the entire $\mathrm{B}-\mathrm{C}$ phase. The mechanical loading is reduced and the membrane relaxes to its initial state with $\lambda=2$. The elastic energy stored in the elastomer gradually decreases until the film returns to its original position. As the area of DE film is reduced, thickness and voltage increase during this process. Due to the presence of Zener diodes, the charge on the DE film is transferred irreversibly to the capacitor. When state $\mathrm{C}$ reached, a residual charge still exists on the $\mathrm{DE}$ film.

In the C-D phase, to harvest the residual charge on the DE film, switch $\mathrm{S}_{2}$ was activated, and the charge in the capacitor and the residual charge on the DE can be harvested by the harvesting circuit. The associated sensor records the amount of charge throughout the charging and discharging process. Due to charge leakage and measurement error of the voltmeter, the amount of charge drawn from power supply is higher than the harvesting charge. Which results in a decrease in energy density.

The achievable energy density depends on how much the capacitance of DE can be changed during an electromechanical cycle. In each generation cycle, the net electric energy harvested is calculated by $E_{\text {net }}=\int \Phi I d t$, where, $I$ is DE current and $\Phi$ is DE voltage. The energy density is defined as the net electrical energy harvested in one entire cycle divided by the mass of DE film. The energy conversion efficiency is defined as net electrical energy divided by the mechanical energy within one electromechanical cycle, which can be expressed as $\eta=E_{\text {net }} / E_{\text {mechanical }} \times 100 \%$.

When the input voltage is $2.2 \mathrm{kV}$ and the pre-stretch ratio is 2, the maximum electrical energy density and energy conversion efficiency in a single harvesting cycle were measured to be $413 \mathrm{~J} / \mathrm{kg}$ and $15.8 \%$, respectively. Under the same conditions, increasing the supply voltage and reducing the minimum pre-stretch ratio will increase the area enclosed by the triangle harvesting schemes in Fig. 1. It is noted that over high supply voltage exceeding the electrical breakdown limit of DE film will lead to the premature failure of the DEG.

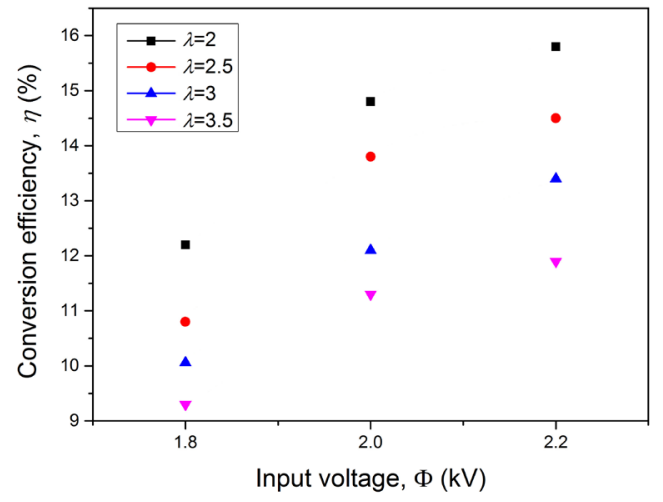

(a)

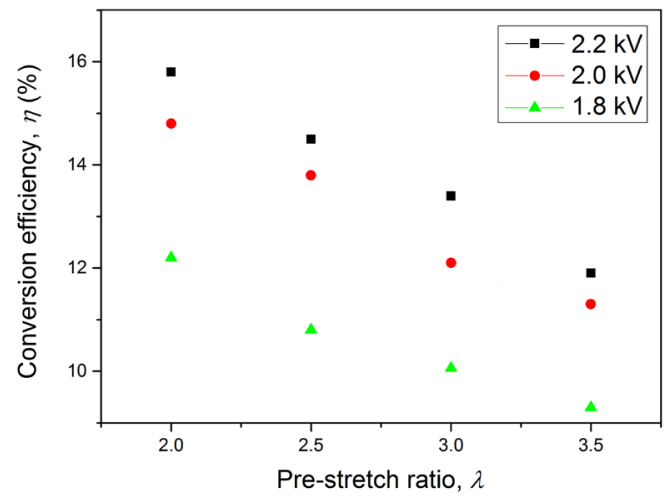

(b)

Fig. 4 (a) Energy conversion efficiency as a function of input voltage,

(b) The energy conversion efficiency as a function of pre-stretch ratio.

\section{Conclusion}

A desirable electric energy harvesting cycle of the DEG should cover this enclosed area A-B-C-A in Fig. 1 as much as possible. The influence of input voltage and the stretch ratio range on the performance of DEGs were investigated using equi-biaxial tension loading mode. Based on the experimental results, to increasing electric energy harvested will effectively improve energy 
conversion efficiency, so it is reasonable to choose a higher input voltage and a larger stretch ratio range in order to elevate the conversion efficiency of the DEG. Compared with the theoretical calculation much effort is still needed to further improve the performance of the DEGs.

\section{References}

[1] S. Samuel, J.S. Huang, S.J. Zhu, and D.R. Clarke, Advanced Materials, 2014 (26) 6617.

[2] P. Brochu, H.F. Li, X.F. Niu, and Q.B. Pei, The International Society for Optical Engineering, 2010 (7642) 76422.

[3] J.S. Huang, S. Samuel, Z.G. Suo, and D.R. Clarke, Advanced Functional Materials, 2013 (23) 5056.

[4] H. Prahlad, R. Kornbluh, R. Pelrine, S. Stanford, J. Eckerle, and S. Oh, Smart materials structures and systems, 2005 (13) 100.

[5] J.Y. Zhou, L.Y. Jiang, and R.E. Khayat, Journal of Applied Physics, 2017 (121) 184102.

[6] S.J.A. Koh, X.H. Zhao, and Z.G. Suo, Materials Research Society, 2009 (1218-Z07)-10.

[7] S.J.A. Koh, C. Keplinger, T. Li, S. Bauer, S. Member, and Z.G. Suo, IEEE/ASME Transactions on Mechatronics, 2011 (16) 33.

[8] X.H. Zhao, Z.G. Suo, Applied Physics Letters, 2007 (91) 061921.

[9] J.S. Huang, S. Shian, Z.G. Suo, and D.R. Clarke, Electroactive Polymer Actuators and Devices, 2013 (8687) 86870Q. 